\title{
AN OPPENHEIM TYPE DETERMINANTAL INEQUALITY FOR THE KHATRI-RAO PRODUCT
}

\author{
YongtaO Li AND LiHUA FENG*
}

Abstract. The Khatri-Rao product is a generalization of the classical Hadamard product for block matrices. In this paper, we give an Oppenheim type determinantal inequality for the Khatri-Rao product of two block positive semidefinite matrices, and then we extend our result to multiple block matrices.

Mathematics subject classification (2020): 15A45, 15A60, 47B65.

Keywords and phrases: Khatri-Rao product, Hadamard product, Oppenheim's inequality, Fischer's inequality.

\section{REFERENCES}

[1] T. ANDO, Inequalities for M-matrices, Linear Multilinear Algebra 8 (1980) 291-316.

[2] S. CHEN, Some determinantal inequalities for Hadamard product of matrices, Linear Algebra Appl. 368 (2003) 99-106.

[3] S. CHEN, Inequalities for M-matrices and inverse M-matrices, Linear Algebra Appl. 426 (2007) 610-618.

[4] S. Dong, Q. LI, Extension of an Oppenheim type determinantal inequality for the block Hadamard product, Math. Inequal. Appl. 23 (2) (2020) 539-545.

[5] X. Fu, Y. LIU, Some determinantal inequalities for Hadamard and Fan products of matrices, J. Inequal. Appl. (2016) 262-269.

[6] M. GÜNTHER, L. KLOTZ, Schur's theorem for a block Hadamard product, Linear Algebra Appl. 437 (2012) 948-956.

[7] R. A. Horn, C. R. Johnson, Matrix Analysis, 2nd ed., Cambridge University Press, 2013.

[8] C. G. Khatri, C. R. RaO, Solutions to some functional equations and their applications to charaterization of probability distributions, Sankhya, 30 (1968) 167-180.

[9] S. KIM, J. KIM, H. LEE, Oppenheim and Schur type inequalities for Khatri-Rao product of positive definite matrices, Kyungpook Math. J. 57 (2017) 641-649.

[10] J. LIU, L. ZHU, Some improvement of Oppenheim's inequality for $M$-matrices, SIAM J. Matrix Anal. Appl. 18 (2) (1997) 305-311.

[11] S. LiU, Matrix results on the Khatri-Rao and Tracy-Singh products, Linear Algebra Appl. 289 (1999) 267-277.

[12] S. LiU, Several inequalities involving Khatri-Rao products of positive semidefinite matrices, Linear Algebra Appl. 354 (2002) 175-186.

[13] S. LiU, G. TRENKLER, Hadamard, Khatri-Rao, Kronecker and other matrix products, Int. J. Inf. Syst. Sci. 4 (2008) 160-177.

[14] M. LiN, An Oppenheim type inequality for a block Hadamard product, Linear Algebra Appl. 452 (2014) $1-6$.

[15] A. OppenheIm, Inequalities connected with definite Hermitian forms, J. London Math. Soc. 5 (1930) $114-119$.

[16] G. P. H. StYAn, Hadamard products and multivariate statistical analysis, Linear Algebra Appl. 6 (1973) 217-240.

[17] Z. YAng, J. LiU, Some results on Oppenheim's inequalities for M-matrices, SIAM J. Matrix Anal. Appl. 21 (3) (2000) 904-912. 
[18] X.-D. Zhang, The equality cases for the inequalities of Fischer, Oppenheim, and Ando for general $M$-matrices, SIAM J. Matrix Anal. Appl. 25 (3) (2004) 752-765.

[19] X.-D. Zhang, C.-X. Ding, The equality cases for the inequalities of Oppenheim and Schur for positive semi-definite matrices, Czechoslovak Math. J. 59 (2009) 197-206.

[20] F. Zhang, Matrix Theory: Basic Results and Techniques, 2nd ed., Springer, New York, 2011. 